\title{
Improvement Generative Growth of Coffea arabica L. Using Plant Growth Regulators and Pruning
}

\author{
Ade Astri Muliasari ${ }^{1, *}$, Ratih Kemala Dewi ${ }^{1}$, Hidayati Fatchur Rochmah ${ }^{1}$, \\ Andoniana Rakoto Malala ${ }^{2,3}$, and Praptiningsih Gamawati Adinurani ${ }^{4}$ \\ ${ }^{1}$ Department of Plantation Production Technology and Management, College of Vocational Studies, \\ Bogor Agricultural University, Jl. Kumbang No. 14, Kampus IPB Cilibende Bogor 16151, \\ West Java, Indonesia \\ ${ }^{2}$ Graduate School of Agricultural Science, Kobe University 1-1 Rokkodai, Nada-ku, \\ Kobe, 657-8501, Japan \\ ${ }^{3}$ Centre de Formation et d'Application du Machinisme Agricole (CFAMA) Route Betafo \\ Ambaniandrefana - B.p. 109, Antsirabe, Madagascar \\ ${ }^{4}$ Department of Agrotechnology, Merdeka University of Madiun, Jl. Serayu No.79, \\ Madiun 63133, East Java, Indonesia
}

\begin{abstract}
Pruning coffee plants is intended to stimulate generative growth that increases productivity to reach optimal. Plant Growth Regulator (PGR) makes it possible to exploit the production potential of plants. Pruning management, combined with the PGR in coffee plants, is expected to improve flowering and berry growth. The research was conducted for 7 mo from October 2017 to April 2018 at Gunung Gede, Bogor, West Java, Indonesia. A split-plot design with two treatment factors was used in this research. Pruning was placed as the main plot consisting of two levels, namely without (P0) and with pruning (P1). The PGR application was placed as a subplot, consisting of three dosages, namely without PGR (Z0), and concentrations of $0.3 \mathrm{~mL} \mathrm{~L}^{-1}$ (Z1) and $0.4 \mathrm{~mL} \mathrm{~L}^{-1}(\mathrm{Z} 2)$. The results showed, the pruning treatment significantly influenced microclimate, number of branches, B0, B1 and B2, number of berry sets, and berries. Pruning treatment had a very significant effect on microclimate, plant height, number of branches, number of coffee fruits, yields $\mathrm{crops}^{-1}$, and productivity. Interaction both significantly affected plant height and $\mathrm{B} 0$. The best dosage had not yet been found.
\end{abstract}

Key words: Branches, coffee arabica, improve coffee flowering and berry growth, increase coffee production

\section{Introduction}

Indonesia is the fourth world coffee exporter after Brazil, Colombia, and Vietnam [1]. Coffee is one of the leading commodities in Indonesia, with positive impacts in socio-economic [2], health science [3, 4], and others. Coffee performance is not only in

\footnotetext{
* Corresponding author: ade.astri@apps.ipb.ac.id
} 
beans. But it is also shown by the side product, among others by solid waste, namely husks and pulp [5-7].

In order to become the largest coffee exporter, Indonesia must increase its coffee yield by developing some technologies. The availability of technologies, such as pruning and plant growth regulators are important. Pruning activities are very important so that coffee plants remain productive continuously [8]. Pruning is useful for breaking the life cycle of pests and diseases, forming a balanced plant canopy between branches, leaves, and fruit production, preventing fruit density, and shooting death. Trimming remnants can be used for soil mulch. Mulching can prevent soil erosion. Decomposed mulch can function as organic fertilizer [8]. In addition, pruning of coffee plants is intended to stimulate generative growth, so that productivity is more optimal and continuous throughout the year.

Plant growth regulators (PGR) in low concentrations can encourage, inhibit, or modify growth and development both in quantity and quality. PGR improves the absorption of nutrients by plants [9], PGR makes it possible to exploit the production potential of plants. In addition, PGR serves to accelerate the response of plants to the environment [10]. Plants can produce natural PGR, but their quantities are limited so that synthetic PGR needs to be added. PGR acts as activators plant cell metabolism, strengthens the immune system, and reacts physiologically. In Coffea arabica L. plants, the number of shoots can be increased with the application of growth regulators and fertilizers [11]. Pruning management, combined with the application of PGR in coffee plants, is expected to improve flowering and berry growth.

\section{Material and methods}

\subsection{Study site and treatments}

The experiment was conducted for 7 mo from October 2017 to April 2018 at Gunung Gede, Bogor, West Java, Indonesia Teaching Farm. The planting material used was the Catimor variety of coffee plants from Catura versus Hibrido De Timor, which was $3 \mathrm{yr}$ old after planting. The growth regulator used was Paclobutrazol. PGR was applied by watering through the ground instead of spraying it directly to the plant parts to reduce the negative impact.

The dosage of fertilizers was listed in Table 1.

Table 1. Fertilizer dosage

\begin{tabular}{|c|c|c|c|c|c|c|c|c|}
\hline $\begin{array}{l}\text { Plant age } \\
\text { (yr) }\end{array}$ & \multicolumn{4}{|c|}{$\begin{array}{l}\text { The early rainy season } \\
\text { (g plant }^{-1} \text { ) }\end{array}$} & \multicolumn{4}{|c|}{$\begin{array}{c}\text { The end of the rainy season } \\
\left(\mathrm{g} \text { plant }{ }^{-1}\right)\end{array}$} \\
\hline & Urea & SP 36 & $\mathrm{KCl}$ & Kieserit & Urea & SP 36 & $\mathrm{KCl}$ & Kieserit \\
\hline 1 & 20 & 25 & 15 & 10 & 20 & 25 & 15 & 10 \\
\hline 2 & 50 & 40 & 40 & 15 & 50 & 40 & 40 & 15 \\
\hline 3 & 75 & 50 & 50 & 25 & 75 & 50 & 50 & 25 \\
\hline 4 & 100 & 50 & 70 & 35 & 100 & 50 & 70 & 35 \\
\hline 5 to 10 & 150 & 80 & 100 & 50 & 150 & 80 & 100 & 50 \\
\hline$>10$ & 200 & 100 & 125 & 70 & 200 & 100 & 125 & 70 \\
\hline
\end{tabular}

\subsection{Trial set up}

A split-plot design with two treatment factors was used in the research. Pruning was placed as the main plot consisting of two levels, namely without pruning (P0) and pruning (P1). The PGR application was placed as a subplot, consisting of three dosages of PGR, namely 
without the application of PGR (Z0), application of concentrations of $0.3 \mathrm{~mL} \mathrm{~L}^{-1}(\mathrm{Z1})$, and $0.4 \mathrm{~mL} \mathrm{~L}^{-1}(\mathrm{Z} 2)$. Therefore, there were six treatment combinations, and each of them consists of three replications. There were 18 experimental units. Each experimental unit consisted of three coffee plants.

The parameters observed were microclimate, such as temperature, humidity. Vegetative growth like plant height, number of branches, and generative growth such as productive branches, number of B0 (branches not yet bearing fruit), B1 (branches that have been fruiting once), B2 (branches that have been fruiting twice), B3 (branches that have been fruiting three times), number of berry sets, number of berries, yields crops ${ }^{-1}$, and productivity.

\section{Results and discussions}

\subsection{Respon of microclimate to pruning treatment}

Microclimate observations were carried out three times in the morning, noon, and afternoon. In general, the temperature begins to increase from morning to afternoon and then decrease in the afternoon. Pruning (P1) treatment has a significant effect on temperature at noon (Table 3) and afternoon (Table 4) but not significantly in the morning (Table 2) around the canopy coffee plant.

Table 2. Effect pruning treatment on the temperature in the morning (at $07.00 \mathrm{am}$ )

\begin{tabular}{|l|c|c|c|c|c|c|}
\hline \multirow{3}{*}{ Treatment } & \multicolumn{7}{|c|}{ Age (months after application) } \\
\cline { 2 - 7 } & 1 & 2 & 3 & 4 & 5 & 6 \\
\cline { 2 - 7 } & \multicolumn{7}{|c|}{ Temperature $\left({ }^{\circ} \mathrm{C}\right)$} \\
\hline Pruning & $26.40 \mathrm{a}$ & $26.24 \mathrm{a}$ & $26.45 \mathrm{a}$ & $27.26 \mathrm{a}$ & $27.40 \mathrm{a}$ & $27.35 \mathrm{a}$ \\
\hline P0 & $26.53 \mathrm{a}$ & $26.62 \mathrm{a}$ & $26.40 \mathrm{a}$ & $27.24 \mathrm{a}$ & $27.30 \mathrm{a}$ & $27.35 \mathrm{a}$ \\
\hline P1 & No & No & No & No & No & No \\
\hline Pr $>$ F & No
\end{tabular}

Description: *: Significant difference $(p<0.05)$, **: Significant difference $(p<0.01)$, No: Not significant, P0: Without pruning, P1: Pruning

Table 3. Effect pruning treatment on the temperature at noon (at 12.00 o'clock)

\begin{tabular}{|l|c|c|c|c|c|c|}
\hline \multirow{2}{*}{ Treatment } & \multicolumn{7}{|c|}{ Age (months after application) } \\
\cline { 2 - 7 } & 1 & 2 & 3 & 4 & 5 & 6 \\
\cline { 2 - 7 } & \multicolumn{7}{|c|}{ Temperature $\left({ }^{\circ} \mathrm{C}\right)$} \\
\hline Pruning & $31.05 \mathrm{a}$ & $30.97 \mathrm{~b}$ & $31.11 \mathrm{a}$ & $31.04 \mathrm{a}$ & $31.21 \mathrm{a}$ \\
\hline P0 & $30.97 \mathrm{~b}$ & $31.31 \mathrm{a}$ & $31.34 \mathrm{a}$ & $31.28 \mathrm{a}$ & $31.35 \mathrm{a}$ & $31.34 \mathrm{a}$ \\
\hline P1 & $31.32 \mathrm{a}$ & $*$ & No & No & No \\
\hline Pr $>$ F & $*$ & No & $*$ & S
\end{tabular}

Description: *: Significant difference $(p<0.05)$, **: Significant difference $(p<0.01)$, No: Not significant, P0: Without pruning, P1: Pruning 
Table 4. Effect pruning treatment on the temperature in the afternoon (at $05.00 \mathrm{pm}$ )

\begin{tabular}{|c|c|c|c|c|c|c|}
\hline \multirow{3}{*}{ Treatment } & \multicolumn{6}{|c|}{ Age (months after application) } \\
\hline & 1 & 2 & 3 & 4 & 5 & 6 \\
\hline & \multicolumn{6}{|c|}{ Temperature $\left({ }^{\circ} \mathrm{C}\right)$} \\
\hline \multicolumn{7}{|l|}{ Pruning } \\
\hline P0 & $26.31 \mathrm{~b}$ & $26.38 \mathrm{~b}$ & $26.44 b$ & $27.53 \mathrm{a}$ & $27.64 \mathrm{a}$ & $27.46 \mathrm{a}$ \\
\hline $\mathrm{P} 1$ & $26.95 \mathrm{a}$ & $26.94 \mathrm{a}$ & $26.97 \mathrm{a}$ & $27.75 \mathrm{a}$ & $27.61 \mathrm{a}$ & $27.84 a$ \\
\hline $\operatorname{Pr}>F$ & $* *$ & $*$ & $*$ & No & No & No \\
\hline
\end{tabular}

Pruning (P1) treatment had a very significant effect on the humidity in the morning (Table 5) at the age of 5 MAP to 6 MAP (month after application) and afternoon (Table 7) at the age of 2 MAP to 4 MAP. At noon (Table 6), pruning treatment had a significant effect on the 4 MAP compared to the treatment without pruning ( $\mathrm{P} 0)$. Humidity began to decrease from morning to noon and increase in the afternoon. Pruning treatment could reduce the moisture, to reduce fruit desiccation.

Pruning techniques could improve air circulation and reduced humidity in the microclimate to reduce disease attacks. On the other hand, pruning will reduce photosynthesis competition between fruit and leaves [12].

Table 5. Effect of pruning treatment on Relative Humidity (RH) in the morning (at $7.00 \mathrm{am}$ )

\begin{tabular}{|l|c|c|c|c|c|c|}
\hline \multirow{3}{*}{ Treatment } & \multicolumn{7}{|c|}{ Age (months after application) } \\
\cline { 2 - 7 } & 1 & 2 & 3 & 4 & 5 & 6 \\
\cline { 2 - 7 } & \multicolumn{7}{|c|}{ Relative humidity (\%) } \\
\hline Pruning & $74.78 \mathrm{a}$ & $76.77 \mathrm{a}$ & $77.40 \mathrm{a}$ & $69.77 \mathrm{a}$ & $69.11 \mathrm{~b}$ & $69.44 \mathrm{~b}$ \\
\hline P0 & $76.88 \mathrm{a}$ & $77.22 \mathrm{a}$ & $78.22 \mathrm{a}$ & $70.22 \mathrm{a}$ & $70.55 \mathrm{a}$ & $70.88 \mathrm{a}$ \\
\hline P1 & No & No & No & No & $* *$ & $* *$ \\
\hline Pr > F &
\end{tabular}

Description: *: Significant difference $(p<0.05)$, **: Significant difference $(p<0.01)$, No: Not significant, P0: Without pruning, P1: Pruning

Table 6. Effect of pruning treatment on RH at noon (at 12.00 o'clock)

\begin{tabular}{|l|c|c|c|c|c|c|}
\hline \multirow{2}{*}{ Treatment } & \multicolumn{7}{|c|}{ Age (months after application) } \\
\cline { 2 - 7 } & 1 & 2 & 3 & 4 & 5 & 6 \\
\hline \multicolumn{7}{|c|}{ Relative humidity (\%) } \\
\hline Pruning & $56.11 \mathrm{a}$ & $57.11 \mathrm{a}$ & $57.11 \mathrm{a}$ & $55.77 \mathrm{~b}$ & $56.44 \mathrm{a}$ & $56.33 \mathrm{a}$ \\
\hline P0 & $56.00 \mathrm{a}$ & $56.66 \mathrm{a}$ & $56.66 \mathrm{a}$ & $58.55 \mathrm{a}$ & $58.22 \mathrm{a}$ & $58.00 \mathrm{a}$ \\
\hline P1 & No & No & No & $*$ & No & No \\
\hline Pr $>$ F & No
\end{tabular}

Description: *: Significant difference $(p<0.05)$, **: Significant difference $(p<0.01)$, No: Not significant, P0: Without pruning, P1: Pruning 
Table 7. Effect of pruning treatment on RH in afternoon (at $05.00 \mathrm{pm}$ )

\begin{tabular}{|c|c|c|c|c|c|c|}
\hline \multirow{3}{*}{ Treatment } & \multicolumn{6}{|c|}{ Age (months after application) } \\
\hline & 1 & 2 & 3 & 4 & 5 & 6 \\
\hline & \multicolumn{6}{|c|}{ Relative humidity (\%) } \\
\hline \multicolumn{7}{|l|}{ Pruning } \\
\hline $\mathrm{P} 0$ & $90.66 \mathrm{a}$ & $91.22 \mathrm{a}$ & $91.44 \mathrm{a}$ & $79.44 \mathrm{~b}$ & $79.15 \mathrm{a}$ & $80.33 a$ \\
\hline $\mathrm{P} 1$ & $87.77 \mathrm{~b}$ & $88.00 \mathrm{~b}$ & $87.88 \mathrm{~b}$ & $80.44 a$ & $79.55 \mathrm{a}$ & $80.53 a$ \\
\hline $\operatorname{Pr}>F$ & $*$ & $* *$ & $* *$ & $* *$ & No & No \\
\hline
\end{tabular}

\subsection{Response of vegetative growth $C$. arabica to pruning and PGR treatment}

Pruning treatment had a very significant effect on plant height at the age of 2 MAP to 6 MAP (Table 8). Pruning treatment produced the lowest plant height and achieved optimal production. In addition, pruning was very useful in facilitating the harvesting of crops. The application of PGR treatment had no effect of planting height from beginning to end observation 0 MAP to 6 MAP. Interaction between pruning and PGR application had a significant effect on the plant height at the age of 2 MAP.

Table 8. Effect of pruning and PGR treatment to plant height

\begin{tabular}{|c|c|c|c|c|c|c|c|}
\hline \multirow{2}{*}{ Treatment } & \multicolumn{7}{|c|}{ Age (months after application) } \\
\hline & 0 & 1 & 2 & 3 & 4 & 5 & 6 \\
\hline & & & &..$(\mathrm{cm})$ & w & & $\ldots$ \\
\hline \multicolumn{8}{|l|}{ Pruning } \\
\hline P0 & $164.37 \mathrm{a}$ & $166.63 \mathrm{a}$ & $177.04 \mathrm{a}$ & $185.44 \mathrm{a}$ & $188.55 \mathrm{a}$ & $178.85 \mathrm{a}$ & $183.96 \mathrm{a}$ \\
\hline P1 & $166.63 \mathrm{a}$ & $164.37 \mathrm{a}$ & $149.00 \mathrm{~b}$ & $149.07 \mathrm{~b}$ & $149.07 b$ & $149.07 b$ & $148.70 \mathrm{~b}$ \\
\hline $\operatorname{Pr}>F$ & No & No & $* *$ & $* *$ & $* *$ & $* *$ & $* *$ \\
\hline \multicolumn{8}{|c|}{ PGR application } \\
\hline $\mathrm{Z} 0$ & $172.22 \mathrm{a}$ & $166.39 \mathrm{a}$ & $173.22 \mathrm{a}$ & $170.27 \mathrm{a}$ & $172.00 \mathrm{a}$ & $167.66 \mathrm{a}$ & $169.72 \mathrm{a}$ \\
\hline $\mathrm{Z1}$ & $159.17 \mathrm{a}$ & $165.11 \mathrm{a}$ & $159.16 \mathrm{a}$ & $170.94 \mathrm{a}$ & $172.55 \mathrm{a}$ & $166.00 \mathrm{a}$ & $169.44 \mathrm{a}$ \\
\hline $\mathrm{Z} 2$ & $164.11 \mathrm{a}$ & $157.56 \mathrm{a}$ & $164.11 \mathrm{a}$ & $160.55 \mathrm{a}$ & $161.89 \mathrm{a}$ & $158.22 \mathrm{a}$ & $159.83 \mathrm{a}$ \\
\hline $\operatorname{Pr}>F$ & No & No & No & No & No & No & No \\
\hline Interaction & No & No & $*$ & No & No & No & No \\
\hline
\end{tabular}

Description: *: Significant difference $(p<0.05)$, **: Significant difference $(p<0.01)$, No: Not significant, P0: Without pruning, P1: Pruning, Z0: $0 \mathrm{~mL} \mathrm{~L}^{-1}, \mathrm{Z1}: 0.3 \mathrm{~mL} \mathrm{~L} \mathrm{~L}^{-1}$, Z2: $0.4 \mathrm{~mL} \mathrm{~L}^{-1}$

Pruning treatment gave a very significant effect on the number of branches at the age of 1 MAP to 6 MAP (Table 9), but the application of PGR treatment did not affect the number of branches. The average number of branches was 49.85 branches on without pruning (P0) and 33.40 branches on pruning treatment (P1) in the last observation.

Pruning treatment stimulated new branches that are continuous in sufficient quantities to support the continuity of production. Pruning coffee plants facilitated the entry of light to stimulate the formation of evenly distributed flower primordia and perfect flowering [13]. The effect of pruning and PGR applications on the number of branches can be seen in Table 9. 
Table 9. Effect of pruning and PGR treatment to the number of branches

\begin{tabular}{|c|c|c|c|c|c|c|c|}
\hline \multirow{3}{*}{ Treatment } & \multicolumn{7}{|c|}{ Age (months after application) } \\
\hline & 0 & 1 & 2 & 3 & 4 & 5 & 6 \\
\hline & \multicolumn{7}{|c|}{ 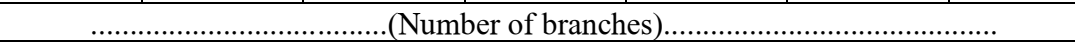 } \\
\hline \multicolumn{8}{|c|}{ 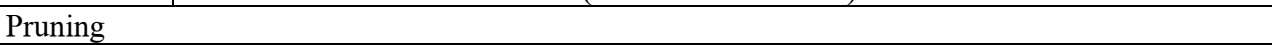 } \\
\hline P0 & $38.370 \mathrm{a}$ & $40.77 \mathrm{a}$ & $41.22 \mathrm{a}$ & $52.96 \mathrm{a}$ & $53.22 \mathrm{a}$ & $46.88 \mathrm{a}$ & $49.85 \mathrm{a}$ \\
\hline P1 & $25.04 \mathrm{a}$ & $26.22 \mathrm{~b}$ & $33.15 \mathrm{~b}$ & $33.77 \mathrm{~b}$ & $33.85 \mathrm{~b}$ & $32.44 \mathrm{~b}$ & $33.40 \mathrm{~b}$ \\
\hline $\operatorname{Pr}>F$ & No & * & $*$ & ** & ** & * & ** \\
\hline \multicolumn{8}{|c|}{ PGR application } \\
\hline $\mathrm{Z} 0$ & $31.33 \mathrm{a}$ & $34.33 \mathrm{a}$ & $38.78 \mathrm{a}$ & $43.72 \mathrm{a}$ & $44.11 \mathrm{a}$ & $39.72 \mathrm{a}$ & $42.27 \mathrm{a}$ \\
\hline $\mathrm{Z1}$ & $30.11 \mathrm{a}$ & $34.77 \mathrm{a}$ & $38.67 \mathrm{a}$ & $44.00 \mathrm{a}$ & $44.11 \mathrm{a}$ & $40.83 \mathrm{a}$ & $42.66 \mathrm{a}$ \\
\hline$\overline{\mathrm{Z} 2}$ & $33.667 \mathrm{a}$ & $34.11 \mathrm{a}$ & $34.11 \mathrm{a}$ & $42.38 \mathrm{a}$ & $42.38 \mathrm{a}$ & $38.44 a$ & $39.94 a$ \\
\hline $\operatorname{Pr}>\mathrm{F}$ & No & No & No & No & No & No & No \\
\hline Interaction & No & No & No & No & No & No & No \\
\hline
\end{tabular}

\subsection{The response of generative growth $C$. arabica to pruning and PGR treatment}

Pruning and PGR application did not affect the productive branches formation. Unproductive branches were pruned so that the nutrients supplied could be channeled to the more productive branches. The effect of pruning and PGR treatment on productive branches were shown in Table 10 .

Table 10. Effect of pruning and PGR treatment to productive branches

\begin{tabular}{|c|c|c|c|c|c|c|c|}
\hline \multirow{3}{*}{ Treatment } & \multicolumn{7}{|c|}{ Age (months after application) } \\
\hline & 0 & 1 & 2 & 3 & 4 & 5 & 6 \\
\hline & \multicolumn{7}{|c|}{.......(Productive branches)..... } \\
\hline \multicolumn{8}{|l|}{ Pruning } \\
\hline $\mathrm{P} 0$ & $23.51 \mathrm{a}$ & $23.70 \mathrm{a}$ & $23.77 \mathrm{a}$ & $24.51 \mathrm{a}$ & $24.51 \mathrm{a}$ & $23.77 \mathrm{a}$ & $24.18 \mathrm{a}$ \\
\hline $\mathrm{P} 1$ & $18.88 \mathrm{a}$ & $23.55 \mathrm{a}$ & $23.59 \mathrm{a}$ & $23.00 \mathrm{a}$ & $22.59 \mathrm{a}$ & $23.18 \mathrm{a}$ & $23.81 \mathrm{a}$ \\
\hline $\operatorname{Pr}>F$ & No & No & No & No & No & No & No \\
\hline \multicolumn{8}{|c|}{ PGR application } \\
\hline $\mathrm{Z} 0$ & $22.611 \mathrm{a}$ & $23.22 \mathrm{a}$ & $24.111 \mathrm{a}$ & $23.27 \mathrm{a}$ & $22.94 \mathrm{a}$ & $22.61 \mathrm{a}$ & $23.50 \mathrm{a}$ \\
\hline $\mathrm{Z1}$ & $22.611 \mathrm{a}$ & $23.27 \mathrm{a}$ & $23.500 \mathrm{a}$ & $24.16 \mathrm{a}$ & $24.16 \mathrm{a}$ & $24.55 \mathrm{a}$ & $25.00 \mathrm{a}$ \\
\hline $\mathrm{Z} 2$ & $18.778 \mathrm{a}$ & $20.27 \mathrm{a}$ & $23.278 \mathrm{a}$ & $23.83 \mathrm{a}$ & $23.55 \mathrm{a}$ & $23.27 \mathrm{a}$ & $23.50 \mathrm{a}$ \\
\hline $\operatorname{Pr}>F$ & No & No & No & No & No & No & No \\
\hline Interaction & No & No & No & No & No & No & No \\
\hline
\end{tabular}

Description: *: Significant difference $(p<0.05)$, **: Significant difference $(p<0.01)$, No: Not significant, P0: Without pruning, P1: Pruning, Z0: $0 \mathrm{~mL} \mathrm{~L}^{-1}, \mathrm{Z1}: 0.3 \mathrm{~mL} \mathrm{~L} \mathrm{~L}^{-1}$, Z2: $0.4 \mathrm{~mL} \mathrm{~L}^{-1}$

The effect of pruning showed a significant effect on the amount of B0 at 1 MAP (Table 11). The number of B0 reached 16.85 branches in the treatment without pruning while in the treatment of pruning around 12.74 branches. The PGR treatment did not seem to affect the amount of $\mathrm{B} 0$ from the beginning to the end of the observation. Interaction of pruning treatment and PGR were evident at 2 MAP. 
Table 11. Effect of pruning and PGR treatment to the number of B0

\begin{tabular}{|c|c|c|c|c|c|c|}
\hline \multirow{3}{*}{ Treatment } & \multicolumn{6}{|c|}{ Age (months after application) } \\
\hline & 1 & 2 & 3 & 4 & 5 & 6 \\
\hline & \multicolumn{6}{|c|}{ 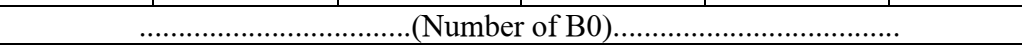 } \\
\hline \multicolumn{7}{|l|}{ Pruning } \\
\hline $\mathrm{P} 0$ & $16.85 \mathrm{a}$ & $11.11 \mathrm{a}$ & $9.98 \mathrm{a}$ & $10.37 \mathrm{a}$ & $17.03 \mathrm{a}$ & $9.51 \mathrm{a}$ \\
\hline $\mathrm{P} 1$ & $12.74 \mathrm{~b}$ & $10.63 \mathrm{a}$ & $8.45 \mathrm{a}$ & $9.51 \mathrm{a}$ & $12.18 \mathrm{a}$ & $7.88 \mathrm{a}$ \\
\hline $\operatorname{Pr}>F$ & * & No & No & No & No & No \\
\hline \multicolumn{7}{|c|}{ PGR application } \\
\hline $\mathrm{Z} 0$ & $15.16 \mathrm{a}$ & $9.61 \mathrm{a}$ & $8.92 \mathrm{a}$ & $9.00 \mathrm{a}$ & $15.66 \mathrm{a}$ & $6.72 \mathrm{a}$ \\
\hline $\mathrm{Z1}$ & $14.72 \mathrm{a}$ & $8.66 \mathrm{a}$ & $8.46 \mathrm{a}$ & $10.11 \mathrm{a}$ & $14.05 \mathrm{a}$ & $9.22 \mathrm{a}$ \\
\hline $\mathrm{Z} 2$ & $14.50 \mathrm{a}$ & $14.33 \mathrm{a}$ & $10.27 \mathrm{a}$ & $10.72 \mathrm{a}$ & $14.11 \mathrm{a}$ & $6.72 \mathrm{a}$ \\
\hline $\operatorname{Pr}>\mathrm{F}$ & No & No & No & No & No & No \\
\hline Interaction & No & * & No & No & No & No \\
\hline
\end{tabular}

Pruning (P1) treatment had a very significant effect (Table 12) on yields (g plant $\left.{ }^{-1}\right)$, fresh and dry fruit yields $\mathrm{ha}^{-1}$ at the second harvest compared to without pruning (P0). The yield plants ${ }^{-1}$ (Figure 1) in pruning treatment reached $1668.36 \mathrm{~g}$ while without pruning only $958.56 \mathrm{~g}$. The importance of pruning activities was to avoid cases of overbearing dieback, namely the death of coffee plants after experiencing heavy growth without being followed by proper maintenance, for example, fertilization and pruning [14]. The application of PGR and their interaction did not significantly affect the yield. Different from the result of [15], the application of a plant growth regulator increased at the number of internodes, the average number of fruits on the fourth and fifth node, and the length of reproductive branches. The productivity of coffee could be increased with the use of plant growth regulators [16].

Table 12. Effect of pruning and PGR treatment on C. arabica yields in the first and second harvest

\begin{tabular}{|l|c|c|c|c|c|c|}
\hline $\begin{array}{c}\text { Treatment } \\
\text { application }\end{array}$ & \multicolumn{2}{|c|}{$\begin{array}{c}\text { Yield } \\
\text { g plant }^{-1}\end{array}$} & \multicolumn{2}{c|}{$\begin{array}{c}\text { Wet fruit yield (berries) } \\
\mathrm{kg} \mathrm{ha}^{-1}\end{array}$} & \multicolumn{2}{c|}{$\begin{array}{c}\text { Dry fruit yield } \\
\text { (productivity) } \\
\mathrm{kg} \mathrm{h}^{-1}\end{array}$} \\
\hline \multicolumn{1}{|c|}{ Pruning } & 1 & 2 & 1 & 2 & 1 & 2 \\
\hline P0 & $2381.88 \mathrm{a}$ & $985.56 \mathrm{~b}$ & $4763.76 \mathrm{a}$ & $1971.12 \mathrm{a}$ & $952.75 \mathrm{a}$ & $394.22 \mathrm{a}$ \\
\hline P1 & $2154.48 \mathrm{a}$ & $1668.36 \mathrm{a}$ & $4308.96 \mathrm{a}$ & $3336.72 \mathrm{a}$ & $861.79 \mathrm{a}$ & $667.34 \mathrm{a}$ \\
\hline Pr $>$ F & No & $* *$ & No & $* *$ & No & $* *$ \\
\hline PGR application & \multicolumn{7}{|l|}{} & & & & \\
\hline Z0 & $2308.44 \mathrm{a}$ & $1526.64 \mathrm{a}$ & $4616.88 \mathrm{a}$ & $3053.28 \mathrm{a}$ & $923.37 \mathrm{a}$ & $610.66 \mathrm{a}$ \\
\hline Z1 & $2454.72 \mathrm{a}$ & $1236.00 \mathrm{a}$ & $4909.44 \mathrm{a}$ & $2472.00 \mathrm{a}$ & $981.88 \mathrm{a}$ & $494.40 \mathrm{a}$ \\
\hline Z2 & $2041.20 \mathrm{a}$ & $1218.24 \mathrm{a}$ & $4082.40 \mathrm{a}$ & $2436.48 \mathrm{a}$ & $816.48 \mathrm{a}$ & $487.29 \mathrm{a}$ \\
\hline Pr $>$ F & No & No & No & No & No & No \\
\hline Interaction & No & No & No & No & No & No \\
\hline
\end{tabular}

Description: *: Significant difference $(p<0.05)$, **: Significant difference $(p<0.01)$, No: Not significant, P0: Without pruning, P1: Pruning, Z0: $0 \mathrm{~mL} \mathrm{~L}^{-1}, \mathrm{Z1}: 0.3 \mathrm{~mL} \mathrm{~L}^{-1}$, Z2: $0.4 \mathrm{~mL} \mathrm{~L}^{-1}$ 


\section{Conclusions}

Pruning treatment significantly influenced microclimate, number of branches, B0, B1 and B2, number of berry sets, and berries. Pruning treatment had a very significant effect on microclimate, plant height, number of branches, number of coffee fruits, yields $\mathrm{crops}^{-1}$, and productivity. Interaction between pruning and PGR significantly affected plant height and B0. The best dosage for PGR treatment had not yet been found.

Thank you very much to Vocational College, Bogor Agricultural University as the sponsor of this research.

\section{References}

1. [ICO] International Coffee Organization. Trade statistics tables. [Online] from http://www.ico.org/prices/m1-exports.pdf (2019). [Accesed on August 3 2020]

2. N. Viartasiwi, A. Trihartono. Coffee Science. 15,e151687:1-5(2020). https://doi.org/10.25186/.v15i.1687

http://www.coffeescience.ufla.br/index.php/Coffeescience/article/view/1687

3. I.D.A.R. Dewanti, P.E. Lestari, R. Budirahardjo, D. Setyorini, R.W.E. Yani, S. Wibisono, et al. Coffee Science. 14,4:477-483(2019).

https://doi.org/10.25186/cs.v14i4.1619

or

http://www.coffeescience.ufla.br/index.php/Coffeescience/article/view/1619

4. I.D.A. Susilawati, S. Suryono, P. Purwanto, J. Burlakovs, A. Yaro. Coffee Science. 15:e151637(2020).

https://doi.org/10.25186/.v15i.1637

or http://www.coffeescience.ufla.br/index.php/Coffeescience/article/view/1637

5. R.H. Setyobudi, S.K. Wahono, P.G. Adinurani, A. Wahyudi, W. Widodo, M. Mel, et al. MATEC Web of Conference. 164,01039:1-13(2018).

https://doi.org/10.1051/matecconf/201816401039

6. R.H. Setyobudi, L. Zalizar, S.K. Wahono, W. Widodo, A. Wahyudi, M. Mel, et al. IOP Conference Series: Earth and Environmental Science. 293,012035: 1-25(2019). https://doi.org/10.1088/1755-1315/293/1/012035 or https://iopscience.iop.org/article/10.1088/1755-1315/293/1/012035/pdf

7. D. Damat, R. Anggriani, R.H. Setyobudi, P. Soni, Coffee Science. 14,4:493-500 (2019). https://doi.org/10.25186/cs.v14i4.1625 http://www.coffeescience.ufla.br/index.php/Coffeescience/article/view/1625

8. W. Rademacher. J. Plant Growth Regul. 34:845-872(2015) https://doi.org/10.1007/s00344-015-9541-6

9. D.C. Baitelle, S.D. Freitas, K.M. Vieira, C.M. Meneghelli, A.C. Verdin-Filho, D.F. Baroni, et al. Journal of Experimental Agriculture International. 21: 1-9(2018) http://www.sciencedomain.org/abstract/23762

10. A. Calatayud, D. Roca, E. Gorbe, P.F. Martines. Scientia Horticulturae. 116: 73-79(2008). https://doi.org/10.1016/j.scienta.2007.10.028

11. R. Cobos, R.M. Mateos, J.M. Alvarez, M.A. Olego, S. Sevillano, S.G. Garcia, et al. Applied and Environmental Microbiology. 81:6474-6483(2015). https://aem.asm.org/content/aem/81/18/6474.full.pdf

12. V.F. Sianturi, A. Wachjar. Bul. Agrohorti. 4,3:266-275(2016). [in Bahasa Indonesia]. https://doi.org/10.29244/agrob.v4i3.14242

13. FAO. Coffee. Institut Africain pour le développement économique et social. B.P. 8008, Abidjan, Côte d'Ivoire (1977). p. 1-69. 
http://www.fao.org/3/ad219e/AD219E06.htm\#ch6.3

14. T. Whelan. Sustainable coffee farming: Improving income and social conditions protecting water, soil and forests. Rainforest Alliance. New York, NY (2014). p. 1-10. https://www.rainforest-alliance.org/sites/default/files/2016-08/sustainablecoffee-farming-report.pdf

15. F.S. Bacilieri, L.C. De Lima, R.M.Q. Lana, D.S.N. Guimaraes, M.A. Clemente. Biosci. J, Uberlandia. 32,2:346-353(2016). https://doi.org/10.14393/BJv32n2a2016-29820

16. P.F.M.C. Filho, D.C. Baitelle, S.J. Freitas, W.S. Silva, P.C. Santos, W.P. Rodrigues. American Journal of Plant Sciences. 9:628-636(2018)

https://doi.org/10.4236/ajps.2018.94049 